\title{
Situação atual da triagem neonatal para hipotireoidismo congênito: críticas e perspectivas
}

\author{
Current situation of neonatal screening for congenital \\ hypothyroidism: criticisms and perspectives
}

Marilza Leal Nascimento'

1 Hospital Infantil Joana de Gusmão, Serviço de Endocrinologia Pediátrica; Universidade Federal de Santa Catarina (UFSC), Departamento de Pediatria, Florianópolis, SC, Brasil
Correspondência para: Marilza Leal Nascimento Rua Rui Barbosa, 152 88025-301 - Florianópolis, SC Brasil

marilzaleal@brturbo.com.br

Recebido em 15/Set/2011 Aceito em 16/Out/2011

\begin{abstract}
SUMÁRIO
O hipotireoidismo congênito $(\mathrm{HC})$ é uma das causas mais frequentes de deficiência mental passível de prevenção. Esforços devem ser utilizados na sua detecção e no tratamento precoces. $\mathrm{O}$ atraso no diagnóstico e no tratamento resultará em sequela neurocognitiva. A triagem neonatal mudou a evolução natural dessa enfermidade. O nível de corte doTSH utilizado é $10 \mathrm{mUI} / \mathrm{l}$. No Brasil, a triagem neonatal é realizada há três décadas. Atualmente todos os estados brasileiros e o Distrito Federal a realizam. Analisando os últimos dados do Programa Nacional de Triagem Neonatal (PNTN), observamos que existe uma diferença enorme entre os Serviços de Referência nos vários estados. A cobertura do PNTN é de $81,61 \%$ dos recém-nascidos. Apenas 56,94\% colheram a amostra até sete dias de vida. Os tempos médios da coleta até a chegada da amostra ao laboratório, da realização da dosagem do TSH, da liberação do resultado e reconvocação das crianças suspeitas estão fora do preconizado, culminando numa idade média de início de tratamento muito acima da ideal. Isso resulta na impossibilidade de cumprimento do principal objetivo da triagem, que é o início precoce do tratamento para a prevenção de sequelas. Estudos recentes têm sugerido mudança do nível de corte doTSH para $6 \mathrm{mUI} / /$ para reduzir os falso-negativos. Medidas devem ser adotadas para que os índices ideais do PNTN sejam atingidos. Arq Bras Endocrinol Metab. 2011;55(8):528-33

\section{Descritores}

Triagem neonatal; hipotireoidismo congênito
\end{abstract}

\section{SUMMARY}

Congenital hypothyroidism $(\mathrm{CH})$ is one of the most common treatable causes of mental retardation. Efforts should be done in its early detection and treatment. Delays in diagnosis and treatment will result in impaired neurocognitive outcomes. Neonatal screening changed the natural history of this disease. The cutoff value for TSH is $10 \mathrm{mUI} / \mathrm{L}$. In Brazil, neonatal screening has been done for three decades. Currently, it is performed in all Brazilian States and the Brazilian Federal District. Looking at recent data on the National Program for Neonatal Screening (NPNS) we can see a huge difference in the results among Brazilian States. NPNS involved $81.61 \%$ of the newborns. Only in $56.94 \%$ of the cases, samples were collected from newborns up to 7 days of life. Mean time of collection to arrival of the specimen in the lab, TSH determination, release of results and summoning the patient are far longer than the ideal times, causing a delay in early treatment to prevent neurological sequelae. Recent studies have suggested that changing TSH cutoff values to $6 \mathrm{mUI} / \mathrm{L}$ may reduce false negative results. Strategies should be adopted to achieve the goals established by the NPNS. Arq Bras Endocrinol Metab. 2011;55(8):528-33

\section{Keywords}

Neonatal screening; congenital hypothyroidism
$\mathrm{O}$ hipotireoidismo congênito (HC) ocorre em l: 2.000 a 4.000 nascidos vivos e afeta duas vezes mais o sexo feminino (1-6). É uma das causas mais comuns de deficiência mental passível de prevenção $(1,2,4)$.
Estabelecer o diagnóstico e iniciar o tratamento precocemente é fundamental para prevenir sequelas $(1,6,7)$.

Esse distúrbio endócrino ocorre devido à deficiência dos hormônios tireoidianos, tiroxina (T4) e triiodotiro- 
nina (T3). Esses hormônios estão relacionados com o funcionamento de vários tecidos e ainda possuem papel primordial no crescimento, na maturação e na organogênese do sistema nervoso central (SNC) $(2,6,8)$.

O período crítico de dependência dos hormônios tireoidianos para formação do SNC abrange desde a vida fetal até 2 anos de idade $(9,10)$. As principais consequências da ausência dos hormônios tireoidianos são: retardo mental grave, falência do crescimento e distúrbios neurológicos como ataxia, incoordenação, estrabismo, movimentos coreiformes e perda auditiva neurossensorial $(11,12)$. O diagnóstico e o tratamento precoces de uma criança com HC representam enorme benefício para a família e para comunidade, evitando custos sociais, emocionais e financeiros de um indivíduo com deficiência mental $(2,6,11,13-19)$.

A maioria dos recém-nascidos com HC mostra-se normal ao nascer. O feto hopotireóideo está protegido até certo ponto pela transferência placentária de hormônio tireoidiano materno (T4) para a circulação fetal. Além disso, ocorre aumento das concentrações cerebrais de tiroxina desiodase, enzima que converte o T4 em T3, fazendo com que a produção de T3 cerebral fique próxima do normal, em detrimento de outras estruturas, como o esqueleto, o que determina atraso na maturação óssea $(6,20-22)$.

A observação clínica é de valor limitado na detecção do HC. O quadro clínico se estabelece lentamente, em semanas ou meses, porém a maior parte das manifestações é inespecífica. Por esse motivo, somente 5\% das crianças são diagnosticadas clinicamente no período neonatal $(1,6,20-22)$.

$\mathrm{O}$ diagnóstico deve ser considerado em qualquer lactente que apresente icterícia prolongada, hipotermia transitória, fontanela posterior aumentada, dificuldade de sucção ou dificuldade respiratória às mamadas. Os sinais clássicos desenvolvem-se durante as primeiras semanas após o nascimento. A maior parte dos sinais e sintomas está presente com 6 a 12 semanas, especialmente letargia, obstipação intestinal e hérnia umbilical. $O$ fácies cretinoide e o retardo de crescimento tornam-se, progressivamente, mais óbvios após os primeiros meses de vida $(1,20)$.

A fim de facilitar o diagnóstico e evitar os danos do hipotireoidismo não tratado, foram criados programas de detecção precoce para HC, conhecidos como "teste do pezinho" ou triagem neonatal (1).

No Brasil, a triagem neonatal é realizada há três décadas. Entretanto, somente em 2001, o Ministério da Saú- de implantou o Programa Nacional de Triagem Neonatal (PNTN) pelo Sistema Único de Saúde (SUS), por meio da Portaria GM/MS no 822, de 6 de junho de 2001. O Programa tem como objetivo promover a detecção de doenças congênitas em fase pré-sintomática em todos os nascidos vivos, permitindo o tratamento precoce $(2,23)$.

Todos os estados brasileiros e o Distrito Federal realizam triagem neonatal para HC.

A dosagem de TSH por imunofluorimetria em amostra de sangue em papel-filtro é a rotina recomendada para a triagem neonatal do $\mathrm{HC}(6,13,24,25)$.

Os valores de corte para reconvocar as crianças variam entre os programas. De modo geral, em crianças com mais de 48 horas de vida e valores de TSH neonatal menores que $10 \mathrm{mUI} / 1$ no sangue total, nenhum seguimento é realizado. Resultados de TSH entre 10 e 20 $\mathrm{mUI} / \mathrm{l}$ determinam a solicitação de uma segunda amostra do calcanhar e, na maioria das vezes, esse segundo resultado virá normal. Entretanto, quando o resultado do TSH neonatal for maior que $20 \mathrm{mUI} / \mathrm{l}$, solicita-se que a criança compareça para consulta clínica, e os testes de função tireoidiana deverão ser realizados em amostras de soro. A maioria das crianças com valores de TSH neonatal maior que $20 \mathrm{mUI} / 1$ apresentará a doença $(15,26)$.

$\mathrm{O}$ valor de corte de TSH adotado no PNTN é de $10 \mathrm{mUI} / \mathrm{l}(23)$.

Um estudo no Reino Unido baixou o limite de corte do TSH para $6 \mathrm{mUI} / \mathrm{l}$. Crianças com o primeiro TSH maior que $20 \mathrm{mUI} / 1$ foram encaminhadas diretamente para avaliação médica. As crianças com TSH entre $6 \mathrm{e}$ $20 \mathrm{mUI} / \mathrm{l}$ foram encaminhadas para uma segunda coleta. Nessa segunda amostra, crianças com TSH maior que $6 \mathrm{mUI} / \mathrm{l}$ foram encaminhadas para avaliação médica e laboratorial. Das 67 crianças com TSH entre 6,1 e $10 \mathrm{mUI} / 1$ no teste inicial, quatro delas continuaram com o TSH maior do que $6 \mathrm{mUI} / 1$ na segunda amostra. Duas delas foram diagnosticadas com HC (27).

Em São Paulo, houve diminuição no valor de corte no programa do Hospital das Clínicas da Faculdade de Medicina de Ribeirão Preto da Universidade de São Paulo (HC-FMRP-USP) no período entre maio de 2005 e outubro de 2007 (28). Crianças com TSH menor que $5 \mathrm{mUI} / \mathrm{l}$ foram consideradas normais, entre 5 e $10 \mathrm{mUI} / \mathrm{l}$ foram consideradas borderline, sendo solicitada uma nova amostra em papel-filtro. Se na segunda amostra fossem observados valores maiores que $5 \mathrm{mUI} / \mathrm{l}$, convocava-se a criança para determinação do TSH no soro. Caso o valor de TSH sérico se mostrasse abaixo ou igual a $4 \mathrm{mUI} / 1$, considerava-se normal. Se 
apresentasse valores acima de $4 \mathrm{mUI} / \mathrm{l}$, a criança seria avaliada e acompanhada clinicamente. Das 76.800 crianças avaliadas, sete foram diagnosticadas com hipotireoidismo e receberam tratamento, o que não aconteceria com o nível de corte de $10 \mathrm{mUI} / \mathrm{l}$ (28).

Os programas de triagem neonatal devem cumprir algumas características, dentre elas ter baixa percentagem de reconvocações ou falso-positivos e reduzido número de perdas ou falso-negativos. A utilização do nível de corte do TSH em $10 \mathrm{mUI} / 1$ poderá aumentar o número de perdas ou falso-negativos. Por outro lado, a utilização do nível de corte do TSH em 6 mUI/l possivelmente aumentará o número de reconvocações ou falso-positivos, porém reduziria o número de falso-negativos (26).

A triagem neonatal deve ser realizada 48 horas após o nascimento e até o $5^{\circ}$ dia de vida em recém-nascidos $(\mathrm{RNs})$ a termo, idealmente antes da alta da maternidade $(1,6,16,29)$. No entanto, altas precoces antes de 48 horas podem resultar em falso-positivos. Os testes de triagem para detecção do HC podem mostrar-se alterados mesmo na ausência de disfunção tireoidiana. Isso acontece, em parte, porque as concentrações hormonais podem sofrer elevações fisiológicas transitórias logo após o parto, e coletas precoces do teste de triagem podem acusar resultados falso-positivos (29).

A estratégia de triagem neonatal para HC recomendada para RNs a termo não é tão eficaz para prematuros e RNs gravemente enfermos (30). Não há um consenso na literatura em relação ao momento ideal para a coleta dos testes de triagem em prematuros ou RNs criticamente doentes. Para prematuros e RNs enfermos com hospitalização prolongada, coleta-se uma amostra inicial do $3^{\circ}$ ao $5^{\circ}$ dia de vida e nova amostra com 1 mês de vida, ou na alta hospitalar, o que ocorrer primeiro (31). Quando houver indicação de transfusão de sangue total, os testes de triagem devem ser coletados antes da transfusão (32).

O principal objetivo dos programas de triagem neonatal para HC é o de estabelecer o diagnóstico e iniciar o tratamento precocemente para evitar sequelas, principalmente neurológicas $(1,6,33,34)$. Para alcançar esse objetivo, é importante que existam padrões de tempo precisos para cada etapa do processo de triagem, porque atrasos em uma ou mais etapas levarão a retardos inaceitáveis no tratamento das crianças afetadas (35).

Estudos clínicos têm demonstrado que algumas variáveis influenciam o quociente de inteligência (QI) das crianças com HC: gravidade da doença (baixa concentração de T4 inicial e atraso na maturação esquelética no RN); idade no início do tratamento; dose de levotiroxina e concentrações séricas de T4 durante o primeiro ano de vida $(17,36-41)$.

As crianças que tiveram o tratamento iniciado até três semanas de vida e a maioria daquelas que o tiveram até seis semanas de vida não apresentaram alterações no desenvolvimento neuropsicomotor, exceto os casos de HC mais graves. Nesses casos, quando iniciaram o tratamento até 14 dias de vida não apresentaram diferenças em relação a crianças normais. A partir desse período apresentam diminuição dos escores alcançados em testes de QI e de desenvolvimento (13,36-39,42).

Atualmente, considera-se que a idade ideal para início do tratamento deve ser no máximo de 14 dias, pois, após essa idade, nos casos graves de HC (atireose e disormonogênese grave), já haverá algum dano cerebral. Quando o tratamento é iniciado precocemente, o impacto negativo de HC grave desaparece (11,13,36-39).

A preparação hormonal tireoidiana de escolha para o tratamento é a T4 (levotiroxina), já que a maior parte do hormônio tireoidiano nas células do SNC deriva da conversão local de T4 em T3 $(6,12,20,21,43)$. Somente comprimidos de levotiroxina deverão ser utilizados, uma vez que não existe aprovação de soluções líquidas desse hormônio (11).

A dose preconizada atualmente é de 10 a $15 \mu \mathrm{g} /$ $\mathrm{kg} /$ dia $(6,11-13,21,24,25,36)$. Preocupação com efeitos indesejáveis sobre o SNC pela dose mais elevada utilizada no tratamento é injustificada, pois crianças a termo e saudáveis apresentam hipertiroxinemia transitória $(16,37,38)$. Ao contrário, o subtratamento, esse sim, é lesivo ao SNC $(36,38,39)$.

$\mathrm{O}$ tratamento deve ser monitorado clinicamente e com dosagens de T4 ou T4 livre e TSH duas a quatro semanas após o início do tratamento, a cada 1 a 2 meses no primeiro ano de vida, a cada 2 a 3 meses entre l e 3 anos, e a cada 3 a 12 meses posteriormente. Se a regularidade do tratamento for questionada ou se os valores hormonais forem anormais, o paciente deve ser monitorado mais frequentemente $(6,11,37)$.

Nos primeiros três anos de vida, deve-se manter os valores de T4 e T4 livre na metade superior da normalidade para o método [T4: 10-16 $\mu \mathrm{g} / \mathrm{dL}$ (130-206 nmol/l); T4 livre: 1,4-2,3 ng/dL (18-30 pmol/l)] e o TSH em níveis normais baixos $(0,5-2,0 \mathrm{mUI} / 1)(37)$.

Dentro das características que um bom método de triagem neonatal deve ter, encontram-se: ampla cobertura populacional, e aqui o ideal seria chegar a $100 \%$ dos recém-nascidos vivos (11), baixa porcentagem de reconvo- 
cações ou falso-positivos $(11,12,14,23,42,43)$, reduzido número de perdas ou falso-negativos $(6,33)$, possibilidade de combinação com outros métodos de rastreamento $(24,25)$, disponibilidade de meios técnicos adequados para confirmação diagnóstica e etiológica $(6,22,24,25)$, possibilidade de iniciar o tratamento precocemente e assegurar seguimento dos afetados $(6,24,25,36-38,43)$.

Dentro das fontes de erros em programas de triagem neonatal, encontram-se: a idade dos RNs no momento da coleta da amostra, erros na técnica de obtenção e conservação das amostras, resultados falso-negativos, atrasos no diagnóstico e no início do tratamento e seguimento $(20,37,44)$.

Mesmo quando não exista dúvida a respeito dos objetivos dos programas de triagem, a capacidade de alcançá-los não pode ser tomada como certa. A disponibilidade de um teste não assegura sucesso ao programa, que deve ser constantemente avaliado (5).

O estudo sobre os programas de triagem neonatal da América Latina demonstrou que, no ano de 2005, a cobertura foi de $49,3 \%$, o que implica que 5,7 milhões de recém-nascidos não participaram dos programas. O Brasil foi incluído no grupo dos países que historicamente possuem sistemas de saúde complexos e subdivididos e que o sistema privado participa das atividades sem fazer parte das estatísticas nacionais. Desse grupo também fazem parte a Argentina, com cobertura do programa de triagem neonatal de $64 \%$, e o México com $70 \%$. Chile, Uruguai e Cuba participam do grupo que possui cobertura de aproximadamente $99 \%$ da população (45).

Analisando os últimos dados do PNTN (Tabela 1), podemos observar que existe uma diferença enorme entre os Serviços de Referência em Triagem Neonatal (SRTN) (46).

De acordo com os indicadores de 2009 do PNTN, que completou este ano dez anos de existência, o Brasil possui uma cobertura de $81,61 \%$ (46). Essa poderia atingir quase $100 \%$ dos RNs, pois todos os estados brasileiros e o Distrito Federal possuem SRTN. Essa cobertura menor pode ser justificada em parte pela realização dos testes de triagem neonatal em laboratórios privados e pela inexistência de legislação que obrigue esses laboratórios a notificar o número de exames realizados e alterados. Uma opção para alcançar a meta de $100 \%$ seria a realização da coleta de sangue nas maternidades antes da alta, bem como a obrigatoriedade de notificação de todos os exames realizados por laboratórios particulares.

Em relação à idade da coleta da amostra para a triagem, é considerado como ideal realizar a coleta de san- gue para o teste de triagem neonatal entre dois e quatro dias de vida $(1,6,11)$. Em alguns países em que esse tempo não é possível de ser atingido, é recomendado que a coleta seja feita, preferencialmente, entre três e cinco dias de vida, não devendo ultrapassar sete dias, sabendo-se que esse atraso é prejudicial para o diagnóstico precoce das doenças metabólicas (6); no PNTN essa é a recomendação. Pelos indicadores do PNTN 2009, apenas em $56,94 \%$ dos RNs foi coletada a amostra na primeira semana de vida; com a ocorrência de atrasos nessa etapa, todas as demais ficaram atrasadas, culminando com o início do tratamento em idade inadequada (46). A média da idade de início do tratamento foi de 40 dias de vida, variando de 18 a 97 dias, não sendo atingido o principal objetivo da triagem neonatal, que é o início precoce do tratamento, possibilitando a prevenção de sequelas, principalmente neurológicas.

Para que o PNTN possa atingir seus objetivos, serão necessárias estratégias para agilizar as várias etapas do programa que determinaram o atraso no início do tratamento.

Visando possibilitar que as crianças com $\mathrm{HC}$ iniciem o tratamento na idade ideal e que elas tenham perspectivas de desenvolvimento semelhantes às de uma criança não acometida, sugerimos que a coleta da primeira amostra seja realizada nas maternidades e hospitais, antes da alta hospitalar dos RNs, sempre que possível. Para os que nascem em domicílio, os pais devem ser orientados a procurar as unidades de saúde do município para a coleta da amostra, idealmente até cinco dias de vida. As amostras devem ser enviadas ao laboratório do SRTN diariamente nos dias úteis, não se admitindo esse tempo médio de nove dias, que foi o que ocorreu no PNTN. O laboratório deve realizar a dosagem de TSH em até três dias. No PNTN esse tempo variou de 3 a 54 dias, com média de 11 dias. É necessário que se tenha uma equipe de agentes de saúde responsável pela convocação, reconvocação e localização das crianças. É importante que seja feito, periodicamente, um trabalho de esclarecimento e reorientação nos hospitais, maternidades, unidades de saúde e postos de coleta para aperfeiçoamento dos profissionais responsáveis pela coleta e envio das amostras de sangue, pois estes, constantemente, sofrem rotatividade.

Em relação ao valor de corte, este também deveria ser revisto para que não ocorram falso-negativos. Talvez o ideal seja fixar esse nível em $6 \mathrm{mUI} / \mathrm{l}$, pois, com o limite em $10 \mathrm{mUI} / 1$, deixam-se de diagnosticar alguns casos de HC. Porém, mais estudos devem ser realizados para avaliar o impacto dessa mudança. 
Tabela 1. Indicadores do Programa Nacional de Triagem Neonatal (PNTN) 2009

\begin{tabular}{|c|c|c|c|c|c|c|c|}
\hline UF & $\begin{array}{l}\text { Cobertura } \\
\text { (\%) }\end{array}$ & $\begin{array}{c}\text { Amostras } \\
\text { coletadas com } \\
\text { até } 7 \text { dias de vida } \\
\text { (\%) }\end{array}$ & $\begin{array}{l}\text { Tempo médio da } \\
\text { coleta até a } \\
\text { chegada da } \\
\text { amostra ao } \\
\text { laboratório }\end{array}$ & $\begin{array}{l}\text { Tempo médio da } \\
\text { chegada da } \\
\text { amostra ao } \\
\text { laboratório até } \\
\text { resultado }\end{array}$ & $\begin{array}{l}\text { Tempo médio do } \\
\text { resultado até } \\
\text { retorno/ } \\
\text { reconvocação }\end{array}$ & $\begin{array}{l}\text { HC: média de } \\
\text { idade na } 1^{\mathrm{a}} \\
\text { consulta }\end{array}$ & $\begin{array}{c}\text { HC: } \\
\text { Total de } \\
\text { casos em } \\
\text { acompanhamento }\end{array}$ \\
\hline$A C$ & 61,28 & 43,42 & 08 & 08 & 09 & 35 & 20 \\
\hline $\mathrm{AL}$ & 76,57 & 30,41 & 08 & 09 & 08 & 20 & 72 \\
\hline AM & 60,57 & 3,08 & 08 & 11 & 08 & 57 & 78 \\
\hline AP & 46,36 & 86,71 & 05 & 35 & 08 & 40 & 12 \\
\hline BA & 86,91 & 27,65 & 11 & 06 & 45 & 47 & 682 \\
\hline CE & 80,22 & 33,78 & 30 & 05 & 20 & 60 & 203 \\
\hline DF & 82,37 & 71,00 & 09 & 04 & 01 & 19 & 162 \\
\hline ES & 92,42 & 52,94 & 07 & 06 & 17 & 35 & 168 \\
\hline GO & 89,39 & 68,50 & 06 & 06 & 14 & 31 & 419 \\
\hline MA & 72,52 & 32,28 & 28 & 14 & 50 & 35 & 166 \\
\hline$M G$ & 90,65 & 85,94 & 04 & 04 & 27 & 19 & 1.070 \\
\hline MS & 89,54 & 47,01 & 03 & 04 & 09 & 21 & 70 \\
\hline MT & 79,71 & 39,57 & 10 & 08 & 37 & 73 & 76 \\
\hline PA & 74,77 & 29,42 & 08 & 07 & 24 & 71 & 298 \\
\hline PB & 83,70 & 46,98 & 15 & 15 & 15 & 41 & 55 \\
\hline$P E$ & 62,48 & 20,43 & 07 & 54 & 12 & 64 & 150 \\
\hline $\mathrm{PI}$ & 74,71 & 87,68 & 07 & 10 & 07 & 56 & 70 \\
\hline PR & 107,44 & 91,28 & 05 & 02 & 16 & 33 & 630 \\
\hline RJ & 81,12 & 43,94 & 14 & 42 & 16 & 35 & 874 \\
\hline RN & 76,97 & 20,26 & 10 & 25 & 22 & 97 & 80 \\
\hline RO & 93,82 & 46,78 & 09 & 04 & 05 & 81 & 41 \\
\hline $\mathrm{RR}$ & 62,84 & 40,31 & 07 & 10 & 24 & 21 & 4 \\
\hline $\mathrm{RS}$ & 74,57 & 43,46 & 05 & 03 & 38 & 26 & 447 \\
\hline SC & 84,67 & 79,68 & 04 & 04 & 03 & 18 & 436 \\
\hline SE & 74,29 & 43,55 & 11 & 17 & 26 & 49 & 230 \\
\hline $\mathrm{SP}$ & 82,38 & 80,83 & 07 & 06 & 65 & 27 & 3.668 \\
\hline \multirow[t]{2}{*}{ TO } & 78,42 & 30,32 & 12 & 13 & 69 & 73 & 43 \\
\hline & 81,61 & 56,94 & 09 & 11 & 22 & 40 & 10.224 \\
\hline
\end{tabular}

Fonte: Ministério da Saúde/Coordenação-Geral de Média e Alta Complexidade/ Relatórios Anuais enviados pelos Serviços de Referência em Triagem Neonatal (SRTN) das Secretarias de Saúde dos Estados e Distrito Federal.

Embora a triagem neonatal tenha melhorado significativamente o prognóstico das crianças com HC, medidas eficazes devem ser adotadas para que haja cumprimento das metas dos tempos de coleta, envio das amostras, realização da dosagem laboratorial, localização das crianças afetadas e, principalmente, início do tratamento em tempo hábil, possibilitando um prognóstico satisfatório para essas crianças.

Declaração: os autores declaram não haver conflitos de interesse científico neste estudo.

\section{REFERÊNCIAS}

1. Lafranch S. Treatment and prognosis of congenital hypothyroidism. Disponível em: <http://www.uptodate.com/contents/treat- ment-and-prognosis-of-congenital-hypothyroidism>. Acesso em: 9 maio 2011.

2. Bevenides $\mathrm{AM}$, Lima $\mathrm{CH}$, Rocha CA, Corrêa AR, El Husny AS, Fernandes-Caldato MC. Perfil epidemiológico de portadores de hipotireoidismo congênito. Rev Para Med. 2006;20(3):23-6.

3. Castanet M, Polak M, Bonaiti-Pellie C, Lyonnet S, Czernichow $P$, Leger J. Nineteen years of national screening for congenital hypothyroidism: familial cases with thyroid dysgenesis suggest the involvement of genetic factors. J Clin Endocrinol Metab. 2001;86(5):2009-14.

4. Rovet J. Congenital hypothyroidism: treatment and outcome. Curr Opin Endocrinol Diabetes. 2005;12(1):42-52.

5. Nascimento ML, Pires MMS, Nassar SM, Ruhland L. Avaliação do programa de rastreamento neonatal para hipotireoidismo congênito da Secretaria de Estado da Saúde de Santa Catarina. Arq Bras Endocrinol Metab. 2003;47(1):75-81.

6. American Academy of Pediatrics, Rose SR; Section on Endocrinology and Committee on Genetics, American Thyroid Association, Brown RS; Public Health Committee, Lawson Wilkins Pediatric Endocrine Society, et al. Update of newborn screening and therapy for congenital hypothyroidism. Pediatrics. 2006;117:2290-303. 
7. Hanukoglu A, Perlman K, Shamis I, Brnjac L, Rovet J, Daneman D. Relationship of etiology to treatment in congenital hypothyroidism. J Clin Endocrinol Metab. 2001;86(1):186-91.

8. Ramalho RJR, Valido DP, Oliveira MH. Avaliação do programa de triagem para o hipotireoidismo congênito no estado de Sergipe. Arq Bras Endocrinol Metab. 2000;44(2):157-61.

9. Kempers MJ, Lanting $\mathrm{Cl}$, van Heijst $\mathrm{AF}$, van Trotsenburg AS, Wiedijk BM, de Vijlder JJ, et al. Neonatal screening for congenital hypothyroidism based on thyroxine, thyrotropin, and thyroxine-binding globulin measurement: potentials and pitfalls. J Clin Endocrinol Metab. 2006;91(9):3370-6.

10. Kempers MJ, van der Sluijs Veer L, Nijhuis-van der Sanden RW, Lanting $\mathrm{Cl}$, Kooistra L, Wiedijk BM, et al. Neonatal screening for congenital hypothyroidism in the Netherlands: cognitive and motor outcome at 10 years of age. J Clin Endocrinol Metab. 2007;92(3):919-24.

11. Grüters $\mathrm{A}$, Krude $\mathrm{H}$. Update on the management of congenital hypothyroidism. Horm Res. 2007;68(Suppl 5):107-11.

12. LaFranchi S. Congenital hypothyroidism: etiologies, diagnosis, and management. Thyroid. 1999;9(7):735-40.

13. Nascimento ML, Silva PC, Simoni G, Lobo GS, Souza CD. [Congenital hypothyroidism screening program: preliminary results]. J Pediatr (Rio J). 1997;73(3):176-9.

14. Song SI, Daneman D, Rovet J. The influence of etiology and treatment factors on intellectual outcome in congenital hypothyroidism. J Dev Behav Pediatr. 2001;22(6):376-84.

15. Maciel LM. Hipotireoidismo congênito. In: Socidade Brasileira de Endocrinologia. Projeto Diretrizes 2005;4(18):1-10. Disponível em: <http://www.projetodiretrizes.org.br/4_volume/18-Hipotiroid. pdf.> Acesso em: 23 ago 2011.

16. Maciel LM, Castro AS, Kimura ET, et al. Hipotireoidismo congênito 2010;4(18):1-13. Disponivel em: <http://www.projetodiretrizes. org.br http://www.projetodiretrizes.org.br/-Hipotiroid.pdf>. Acesso em: 23 ago 2011.

17. Olney RS, Grosse SD, Vogt RF Jr. Prevalence of congenital hypothyroidism--current trends and future directions: workshop summary. Pediatrics. 2010;125(Suppl 2):S31-6.

18. Fisher DA, Klein AH. Thyroid development and disorders of thyroid function in the newborn. N Engl J Med. 1981;304:702-12.

19. Klein RZ, Mitchell ML. Hypothyroidism in infants and children. In: Braverman LE, Utiger RD, editors. Werner and Ingbar's the Thyroid. 7th ed. Philadelphia: Lippincott-Raven Publishers; 1996. p. 984-90.

20. Fisher DA. Disorders of the thyroid in the newborn and infant. In: Sperling MA. Pediatric Endocrinology. Philadelphia: W.B. Saunders; 1996. p. 51-70.

21. LaFranchi S. Congenital hypothyroidism: a newborn screening success story? The Endocrinologist. 1994;331:1072-8.

22. Fisher DA. Management of congenital hypothyroidism. J Clin Endocrinol Metab. 1991;72:523-9.

23. Brasil. Ministério da Saúde. Secretaria de Atenção à Saúde. Departamento de Atenção Especializada do Programa Nacional de Triagem Neonatal: oficinas regionais de qualificação da gestão. Brasília: Editora do Ministério da Saúde; 2006.

24. Toublanc JE. Guidelines for neonatal screening programs for congenital hypothyroidism. Acta Paediatr. 1999;88(Suppl 432):13-4.

25. Working Group on Neonatal Screening of the European Society for Paediatric Endocrinology. Revised guidelines for neonatal screening programmes for primary congenital hypothyroidism. Horm Research. 1999;52:49-52.

26. Nascimento ML. Hipotireoidismo congênito em Santa Catarina. In: Medeiros-Neto G, Knobel M, editors. Hipotireoidismo congênito no Brasil - Desafios à busca de soluções. São Paulo: Conectfarma Publicações Científicas Ltda.; 2008. p. 67-70.
27. Korada SM, Pearce M, Ward Platt MP, Avis E, Turner S, Wastell H, et al. Difficulties in selecting an appropriate neonatal thyroid stimulating hormone (TSH) screening threshold. [Internet]. Arch Dis Child. 2010;95(3):169-73. Disponível em: http://www.ncbi.nlm.nih. gov/pubmed/19679574. Acesso em: 6 set. 2010.

28. Maciel LMZ. Diagnóstico: novos valores de corte? In: Neto-Medeiros G, Knobel M, editors. Hipotireoidismo congênito no Brasil - Desafios à busca de soluções. São Paulo: Conectfarma Publicações Científicas Ltda.; 2008. p. 81-5.

29. Lott JA, Sardovia-lyer M, Speakman KS, Lee KK. Age-dependent cutoff values in screening newborns for hypothyroidism. Clin Biochem. 2004;37:791-7.

30. Gruñeiro-Papendieck L, Prieto L, Chiesa A, Bengolea S, Bossi G, Bergadá $C$. Usefulness of thyroxine and free thyroxine filter paper measurements in neonatal screening for congenital hypothyroidism of preterm babies. J Med Screen. 2000;7:78-81.

31. Hyman SJ, Greig F, Holzman I, Patel A, Wallach E, Rapaport R. Late rise of thyroid stimulating hormone in ill newborns. J Pediatr Endocrinol Metab. 2007;20:501-10.

32. Kaye $\mathrm{Cl}$; Committee on Genetics. Introduction to the newborn screening factsheets. Pediatrics. 2006;118:1304-12.

33. LaFranchi S. Congenital hypothyroidism: a newborn screening success story? The Endocrinologist. 1994;331:1072-8.

34. Brook CGD. The consequences of congenital hypothyroidism. Clin Endocrinol. 1995;42:431-2.

35. Simpson N, Randall R, Lenton S, Walker S. Audit of neonatal screening programme for phenylketonuria and congenital hypothyroidismo. Arch Dis Child Fetal Neonatal Ed. 1997;77:228-34.

36. Dubuis JM, Glorieux J, Richer F, Deal CL, Dussault JH, van Vliet G. Outcome of severe congenital hypothyroidism: closing the developmental gap with early high dose levothyroxine treatment. J Clin Endocrinol Metab. 1996;81:222-7.

37. Fisher DA. The importance of early management in optimizing 10 in infants with congenital hypothyroidism. J Peditr. 2000;136:273-4.

38. Bongers-Schokking JJ, Koot HM, Wiersma D, Verkerk PH, de Muinck Keizer-Schrama SM. Influence of timing and dose of thyroid hormone replacement on development in infants with congenital hypothyroidism. J Pediatr. 2000; 136:292.

39. Bongers-Schokking JJ, de Muinck Keizer-Schrama SM. Influence of timing and dose of thyroid hormone replacement on mental, psychomotor, and behavioral development in children with congenital hypothyroidism. J Pediatr. 2005;147:768.

40. Salerno M, Di Maio S, Militerni R, Argenziano A, Valerio G, Tenore A. Prognostic factors in intelectual development at 7 years of age in children with congenital hypothyroidism. J Endocrinol Invest. 1995;18:774-9.

41. Rovet JF, Ehrlich R. Long-term effects of L-thyroxine therapy for congenital hypothyroidism. J Pediatr. 1995;126:380-6.

42. Hrytsiuk I, Gilbert R, Logan S, Pindoria S, Brook CG. Starting dose of levothyroxine for the treatment of congenital hypothyroidism: a systematic review. Arch Pediatr Adolesc Med. 2002;156:485-91.

43. Gruters A, Jenner A, Krude H. Long-term consequences of congenital hypothyroidism in the era of screening programmes. Best Pract Res Clin Endocrinol Metab. 2002;16(2):369-82.

44. Fisher DA. Effectiveness of newborn screening programs for congenital hypothyroidism. Prevalence of missed cases. Pediatr Clin North Am. 1987;34:881-90.

45. Borrajo GJ. Newborn screening in Latin America at the beginning of the 21st century. J Inherit Metab Dis. 2007;30(4):466-81.

46. BRASIL. Ministério da Saúde. Coordenação Geral de Média e Alta Complexidade. Indicadores do PNTN 2009: Relatórios anuais enviados pelas Secretarias de Saúde dos Estados e Distrito Federal. Disponível em: <http://portal.saude.gov.br/portal/arquivos/pdf/relatorio_gestao_sas_2008.pdf>. Acesso em: 2 set. 2011. 